\title{
Phase Contrast Projection Display Using Photopolymer
}

\author{
Mei-Lan Piao, Nam Kim, and Jae-Hyeung Park* \\ School of Electrical \& Computer Engineering, Chungbuk National University, Cheongju, \\ 361-763, Korea
}

(Received October 9, 2008 : revised November 14, 2008 : accepted November 24, 2008)

\begin{abstract}
We propose a phase contrast filter using photopolymer, for the phase contrast projection display. The photopolymer has high photosensitivity such that its optically induced refractive index change has a linear dependency on the illuminating light intensity. We implemented a phase contrast projection display using photopolymer as a phase contrast filter. By controlling the refractive index change of the photopolymer, we successfully convert an input phase image into a high contrast intensity image. We also investigated the effect of the photopolymer illumination condition on the quality of the displayed intensity image. As a projector, we achieved $82 \%$ phase to intensity conversion efficiency, which implies that the proposed method can potentially have much higher light efficiency than conventional projection display.
\end{abstract}

Keywords : Photopolymer, Phase contrast, Projector, High light efficiency

OCIS codes : (350.2460) Filters, interference; (050.5080) Phase shift; (120.2040) Displays; (230.6120) Spatial light modulators

\section{INTRODUCTION}

The phase contrast method is an interferometric phase visualization technique that converts the phase image to the intensity image by introducing a phase shift of the zero frequency Fourier component. A phase modulated light beam is decomposed into Fourier components using a lens. A wave retarder at the Fourier plane shifts the phase of the lowest spatial frequencies relative to the higher frequency components. Interference between frequency components upon recombination by a second lens creates the desired intensity distribution. It has received considerable attention for its promise in applications that include microscopy [1, 2], materials processing [3], and phase only encryption and decryption in optical information systems $[4,5]$. Recently, laser projection display based on the phase contrast method has been reported $[6,7]$. It draws a new attention in the microprojector application for mobile devices due to its superior light efficiency over the conventional amplitude modulation method. As a phase contrast filter, these previous works have used photorefractive crystals [8],

*Corresponding author: jh.park@cbnu.ac.kr
Kerr medium [3], zinc tetraphenylporphyrin [9], or optical disk with a small pit on the center $[6,7]$.

Photopolymer is a useful medium that exhibits optically induced refractive index change. Photopolymer is easy to fabricate, low cost, and highly sensitive. Due to these advantages, the photopolymer has been used in wide range of the research fields including holographic security system, holographic storage, optical waveguides, and so on.

In this paper, we propose the phase contrast projection display using the photopolymer as a phase contrast filter. The phase contrast filter is implemented by inducing the refractive index change at the central region of the photopolymer. The condition for inducing desired refractive index change is investigated based on the characteristics of the photopolymer. The phase image, which is displayed on the phase-type spatial light modulator (SLM), is converted into high intensity image by the phase contrast filter, and finally captured by CCD camera.

One unique feature of the proposed phase contrast projection display is the use of the photopolymer. The previous works on the phase contrast projection display used the optical disk with a small pit on the center $[6$, 7]. Besides the general advantage of the photopolymer, 
the particular advantage of the photopolymer over the optical disk in the phase contrast projection display system is its self-alignment feature. The optical disk has to be fabricated before it is mounted and aligned in the projection system. Considering that the misalignment of the zero-frequency part (small pit on the optical disk) causes serious degradation in the output intensity image, the alignment process should be done with very high precision. In the photopolymer case, however, the photopolymer can be fabricated into the phase contrast filter after it is mounted in the system. Therefore the necessity for the alignment process is eliminated with the use of the photopolymer. Also, the refractive index change is easily controllable in the photopolymer case, while it is fixed in the optical disk case. This feature makes it possible to find the optimum refractive index change and the corresponding illumination condition more easily.

The use of the photopolymer as a phase contrast filter is first reported by M. L. Piao et al. [10]. However, it was just a proof-of-concept of the possibility of using the photopolymer as a phase contrast filter, and not for the specific application for the projection display. In this paper, we applied the phase contrast technique using photopolymer as a filter to phase contrast projection display with detailed analysis. We investigated the effect of the photopolymer illumination condition on the quality of the displayed intensity image. The experiments were carried out for different beam intensities, exposure times, and illumination energies. From the analysis of the output intensity images, we demonstrated that the illuminating beam intensity and the exposure time in the fabrication process of the phase contrast filter using the photopolymer are the critical parameters for the optimal performance.

\section{CONCEPT OF PHASE CONTRAST PROJECTION DISPLAY}

The concept of phase contrast projection display system is illustrated in Fig. 1.

The phase contrast projection display is basically a 4-f system with the phase contrast filter at the Fourier plane. The phase image at the input plane is Fourier transformed by the first lens. The phase contrast filter provides phase difference between the zero frequency component and higher frequency components. This phase shifted Fourier components are recombined by the second lens, resulting in the intensity image at the output plane.

As a projector, the phase contrast projector has higher light efficiency than the conventional amplitude modulating projector. The amplitude modulating projector displays the image by partially transmitting the light. Therefore a considerable amount of incoming light is

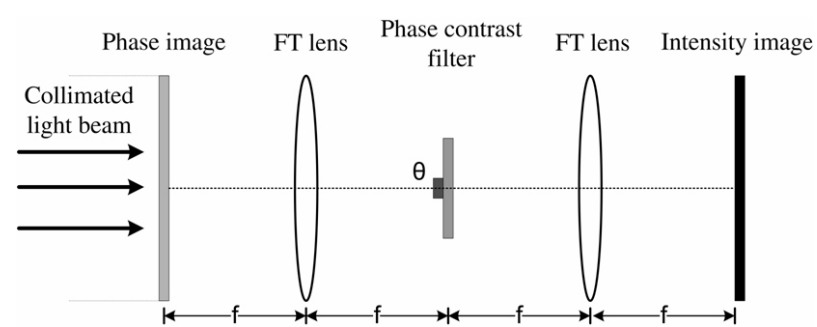

FIG. 1. 4-f setup for phase contrast imaging.

blocked, resulting in low light efficiency. By contrast, the phase contrast projector modulates only the phase of the light and converts it to intensity image using phase contrast filter. Since all incoming light is transmitted and contributes to the formation of the desired image, the light efficiency can be much higher than the amplitude modulating projector.

A digital holographic projection display also uses phase modulation for displaying target intensity image. Heavy computation load, however, is an issue of the digital holographic projection display [11]. In the digital holographic display, the holographic pattern should be computed for the given target image, and this usually involves a huge amount of data, increasing the computation time. On the contrary, for phase contrast projection display, the target image is just displayed in the phase-type SLM without any computation. Therefore, computation time is one advantage of the phase contrast projection display over the digital holographic projection display. Also, Palima et al. showed that the light efficiency of the phase contrast projection display is superior to the digital holographic projection display [12]. Therefore the phase contrast projection display has high potential in the energy saving field.

\section{CHARACTERISTIC OF PHOTOPOLYMER AS PHASE CONTRAST FILTER}

In our work, the photopolymer is used for the phase contrast filter. The photopolymer provides the phase shift by the refractive index modulation, which we can control optically. It is necessary to understand the characteristic of the photopolymer for realizing the desired amount of the modulation of the refractive index. The photopolymer material, which we used as phase contrast filter, typically consists of polymeric binders, monomers, and plasticizer, along with initiating systems including photoinitiators, chain transfer agents, and sensitizing dyes. The monomer and binder affects the physical properties of the film and the magnitude of the index modulation recorded in the film. Refractive index modulation is achieved by monomer diffusion [13]. As a phase contrast filter, the phase shift between zero spatial frequency component and the higher spatial 
frequency components is produced by photo-induced refractive-index change in the photopolymer. The phase shift $\theta$ is given by

$$
\theta=\frac{2 \pi \cdot \Delta n \cdot d}{\lambda}
$$

where $\lambda$ is the wavelength, $\Delta n$ is the refractive-index difference between the sites of the zero order frequency component and the higher order frequency component on the photopolymer, and $d$ is the thickness of photopolymer [9].

In order to get a desired phase shift $\theta$, we need to generate proper refractive index difference $\Delta n$ by illuminating the central part, which is the zero frequency component site of the photopolymer according to Eq. (1). The condition for generating proper refractive index difference $\Delta n$ was investigated by forming the grating in the photopolymer under various experimental conditions. The induced refractive index change $\Delta n$ is monitored by measuring diffraction efficiency of the grating, $\eta$. From Kogelnik's coupled-wave theory [14-16], $\Delta n$ is calculated by

$$
\Delta n=\frac{\lambda \cos \theta_{r}}{\pi d} \sin ^{-1} \sqrt{\eta}
$$

where $\theta_{r}$ is the Bragg incidence angle.

Figure 2 sketches the diffraction efficiency of the gratings. Gratings were recorded with the beam intensity values of $I=0.51,0.74,0.98,1.25,1.78$, and 2.23 $\mathrm{mW} / \mathrm{cm}^{2}$. First, there is an induction time period, in which the material does not respond to the recording energy. Next, grating formation occurs, in which the diffraction efficiency grows until a saturation value is reached. Finally, after saturation, all the monomers are polymerized, and the diffraction efficiency value shows

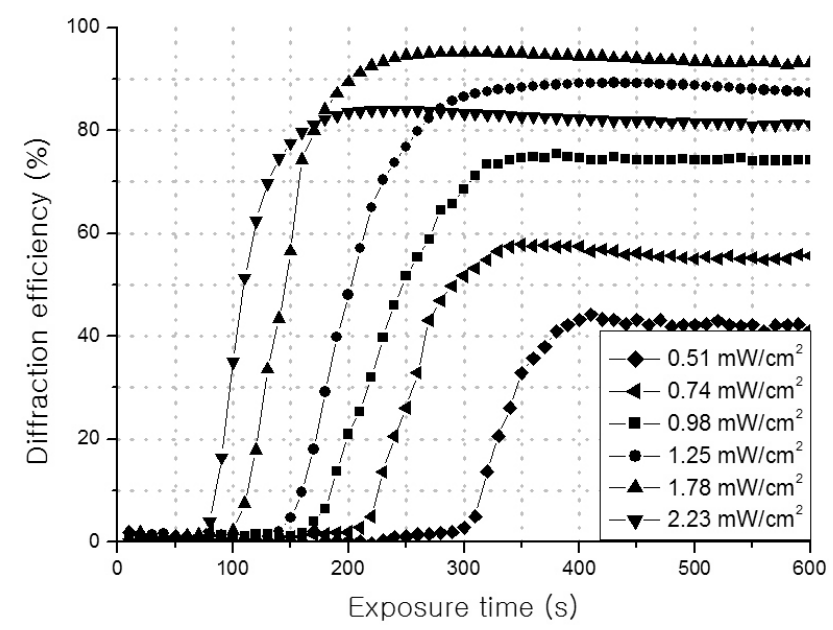

FIG. 2. Diffraction efficiency of the grating recorded in photopolymer. almost no more changes from the saturation value. For photopolymer, the saturation values of the diffraction efficiency are $42,55,74,88,95$, and $81 \%$ for each beam intensity value. The reason of the variation of the saturation values is that when the intensity is high, the polymerization happens so fast that the monomers are polymerized before they can reach the monomer depleted region. As a result, the refractive index difference is small. In the case of the sufficiently low beam intensity, the diffusion time is much shorter than the polymerization time. The monomers are free to move to the region that has lower monomer concentration, and they are polymerized there. As a result, the refractive index modulation can be as high as a maximum available value, which is specific for the material.

For a desired phase shift $\theta$, the corresponding refractive index $\Delta n$ is calculated by Eq. (1). The condition for realizing the corresponding refractive index change $\Delta n$ can be found from Eq. (2) and the photopolymer characteristics shown in Fig. 2. For example, if we want to implement a phase contrast filter of $\frac{\pi}{2}$ phase shift, we can know that the corresponding refractive index change $\Delta n$ is 0.0035 from Eq. (1). Since the grating exhibits $55 \%$ diffraction efficiency with $\Delta n=0.0035$ from Eq. (2), we finally get the beam intensity and exposure time condition which are necessary for $\frac{\pi}{2}$ phase shift by finding the condition for $55 \%$ diffraction efficiency in Fig. 2.

Since the fabrication of the phase contrast filter is achieved by illuminating the central part, i.e. zero frequency part, of the photopolymer, the refractive index profile around the zero frequency area may not be as abrupt as the pre-manufactured optical disk with central pit $[6,7]$, but can have smoothed boundary if the illuminating beam intensity profile is not abrupt. In order to verify its effect on the resultant output intensity image, we performed a simulation for the square shaped refractive index profile and Gaussian shaped refractive index profile. The full-width-at-half-maximum(FWHM) of the Gaussian profile is set to the width of the square profile. Figure 3 is the simulation result. If we define the correlation coefficient between the target image $f$ and the output intensity image $h$ as

$$
\rho=\left|\frac{E\{[|f|-E(|f|)][|h|-E(|h|)]\}}{\left\{E\left\{[|f|-E(|f|)]^{2} E\left\{[|h|-E(|h|)]^{2}\right\}\right\}^{\frac{1}{2}}\right.}\right|
$$

where $E[$ denotes the expected value operator, then the correlation coefficients are calculated to be 0.7858 for the square profile case and 0.7408 for the Gaussian profile case. Visual inspection of the output intensity images shown in Fig. 3 and the correlation coefficient 


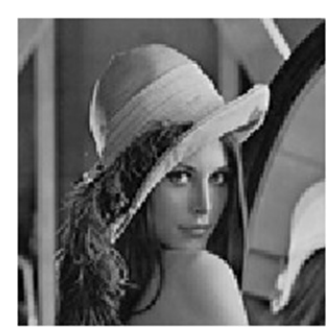

Target image

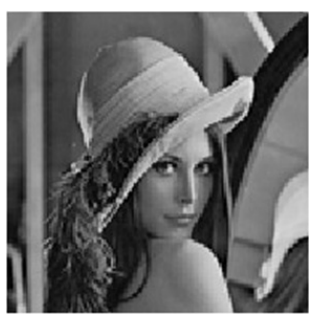

Target image

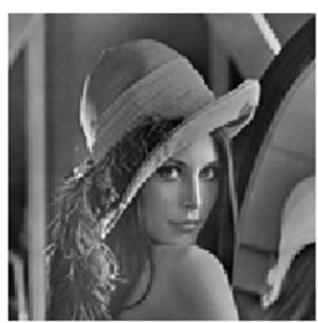

Output intensity image

(a)

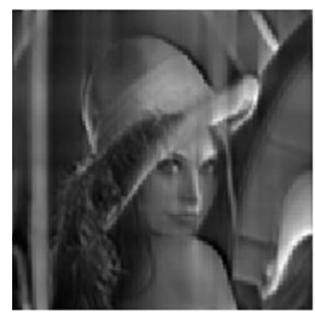

Output intensity image

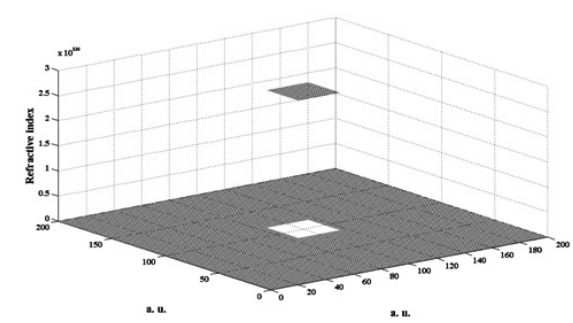

Photopolymer refractive index profile

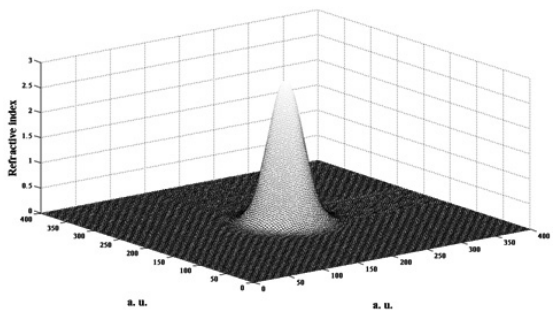

Photopolymer refractive index profile

(b)

FIG. 3. Simulation results. (a) Output intensity image when the photopolymer has abrupt square shape refractive index profile. (b) Output intensity image when the photopolymer has Gaussian shape refractive index profile.

values indicate that the Gaussian shaped refractive index profile slightly deteriorates the output intensity image but its effect is not significant. Therefore, it is desirable to illuminate the photopolymer with a beam of the abrupt intensity profile in the phase contrast filter fabrication but it does not have critical impact on the output intensity image.

\section{EXPERIMENTAL RESULTS}

The experimental setup for the demonstration of the phase contrast projection display system is shown in Fig. 4.

The operating wavelength is $532 \mathrm{~nm}$. The photopolymer used in the experiment is Dupont photopolymer HFR150-38. Our experiment was performed in two steps. First, before we set the phase image on the SLM, we have to obtain the phase shifted photopolymer that is placed at the Fourier plane of the 4-f imaging configuration. Although the optimum phase shift of the phase contrast filter can vary according to the input phase image, it is known that $\frac{\pi}{2}$ phase shift generally provides highly contrasted image when the input phase range is not large $[2,8]$. In the experiment, we implemented $\frac{\pi}{2}$ phase shift on the photopolymer as usual phase contrast filter. Figure 4(a) shows the process of making $\frac{\pi}{2}$ phase shift in photopolymer. The expanded laser beam is reflected by the SLM without any phase modulation and focused on the central part of the photopolymer, inducing the refractive index change. The beam intensity and the exposure time are determined to be $0.74 \mathrm{~mW} / \mathrm{cm}^{2}$ and 8 minutes, respectively as explained in the previous section. The diameter of focused beam size in Fig. 4(a) is approximately $0.2 \mathrm{~mm}$. The second step is the phase contrast projection display with the implemented phase contrast filter. Figure 4(b) shows the setup of the experiment. At this time, the SLM modulate the phase according to the gray value of the desired image. The beam intensity is fixed at $4.44 \mu \mathrm{W} / \mathrm{cm}^{2}$ to prevent any further phase shift in the photopolymer. A half-wave plate and polarizer are used to obtain $+45^{\circ}$ polarized beams. In the setup, a Holoeye LC-R 1080 having twist-aligned liquid-crystal was used as a SLM. This is an electrically addressed SLM that can produce above $1.2 \pi$ phase modulation at a wavelength of $532 \mathrm{~nm}$. The SLM is controlled by digital video interactive (DVI) signal from a computer. With the large pitch size 8.1 $\mu \mathrm{m}$, the fill factor of $90 \%$, and the high resolution $1920 \times 1200$, the SLM provides a large active area 16.39 $\times 10.56 \mathrm{~mm}$. Two lenses in Fig. 4(b) have the focal length $250 \mathrm{~mm}$ for L2 and $200 \mathrm{~mm}$ for L3. The intensitysensitive device located in the output plane is a Kodak MegaPlus Es 1.0 CCD camera with a small pitch size of $9 \mu \mathrm{m}$ and the high resolution $1018 \times 1008$, so that the intensity image can be captured.

Experimental results are shown in Fig. 5.

Figure 5(a) shows the target image to be displayed, Fig. 5(b) is the intensity image captured by the CCD without the photopolymer and Fig. 5(c) is the intensity image captured by the CCD with the photopolymer at 


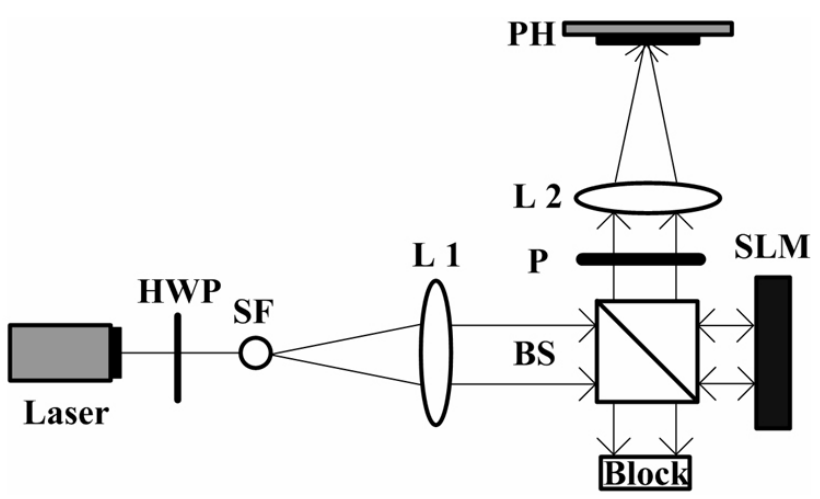

(a)

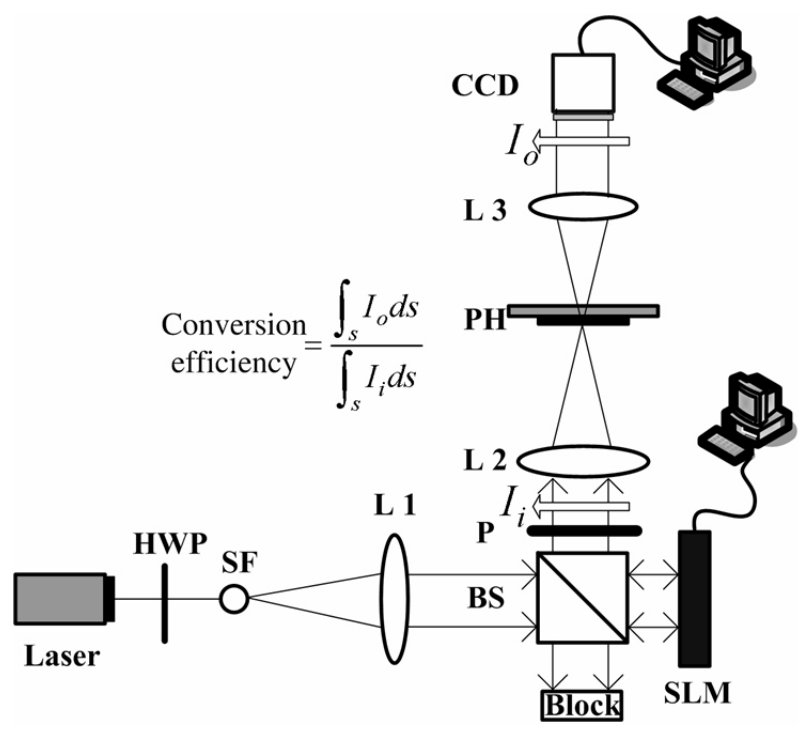

(b)

FIG. 4. (a) Experimental setup for making phase shift in photopolymer. (b) The experimental setup for phase contrast imaging using obtained photopolymer. HWP: Half wave plate; SF: Spatial filter; A: Aperture; BS: Beam splitter; P: Polarizer; L\#: Lenses; PH: Photopolymer; SLM: Spatial light modulator; CCD: Charge coupled device.

the Fourier plane. From Figs. 5(b) and 5(c), we can see that the photopolymer enhances the contrast significantly by the phase contrast image principle. The residual contrast in Fig. 5(b) originates from the undesired amplitude modulation accompanied by the phase modulation of the SLM. With the use of the ideal phase only SLM, this residual contrast is expected to be eliminated, consequently from Fig. 5, we can be confirmed that the phase contrast projection display can be implemented using the phase shifted photopolymer.

The variation of the output intensity image quality according to different photopolymer conditions are shown in Figs. 6, 7 and 9.

Figures 6(a)-6(e) show the output intensity images with arbitrarily chosen different photopolymer conditions. In the proposed method, the phase contrast filter is

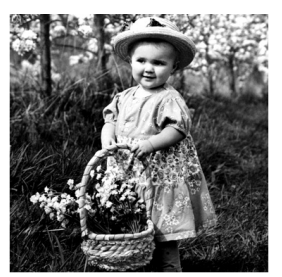

(a)

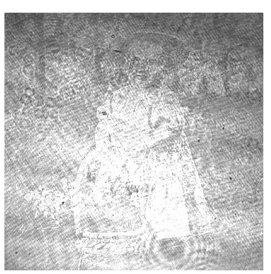

(b)

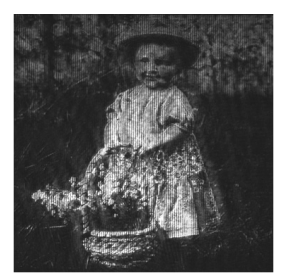

(c)
FIG. 5. Experimental results. (a) Original image to be displayed. (b) Output intensity image without the photopolymer. (c) Output intensity image with the phase shifted photopolymer.

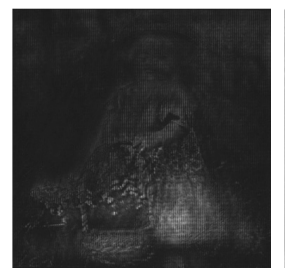

(a)

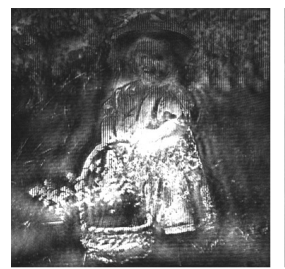

(d)

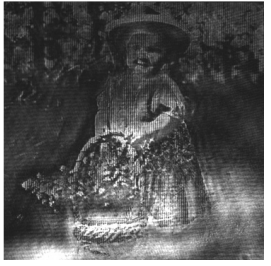

(b)

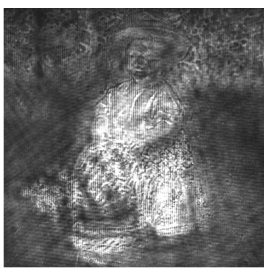

(e)

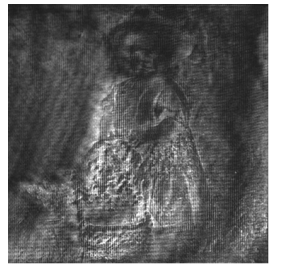

(c)
FIG. 6. Experimental results. Output intensity image with the phase shifted photopolymer corresponding to the five different conditions that (a) $I=0.51 \mathrm{~mW} / \mathrm{cm}^{2}$ and 8.5 minutes exposure time which generates $\Delta n=0.003$, (b) $I=$ $0.98 \mathrm{~mW} / \mathrm{cm}^{2}$ and 8.5 minutes exposure time which generates $\Delta n=0.0043$, (c) $I=1.25 \mathrm{~mW} / \mathrm{cm}^{2}$ and 8.5 minutes exposure time which generates $\Delta n=0.0051$, (d) $I=1.78 \mathrm{~mW} / \mathrm{cm}^{2}$ and 7 minutes exposure time which generates $\Delta n=0.0057$, and (e) $I=2.23 \mathrm{~mW} / \mathrm{cm}^{2}$ and 6 minutes exposure time which generates $\Delta n=0.0047$.

implemented by illuminating the central part of the photopolymer inducing the desired phase shift, or equivalently desired refractive index change $\Delta n$, using the setup shown in Fig. 4(a). If the illuminating beam intensity and the exposure time are not optimized according to the explanation in the previous section and the measured photopolymer characteristics shown in Fig. 2, the desired $\Delta n$ will not be achieved, degrading the phase contrast imaging performance. This argument is confirmed by Fig. 6. We can observe that only when the illuminating condition is optimal, the output image has high contrast, showing optimal performance.

Figure 7(a)-7(f) show the output intensity images when the refractive index change of the photopolymer is generated by the illumination of the same energy but different beam intensities and exposure times. For 


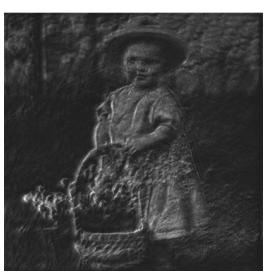

(a)

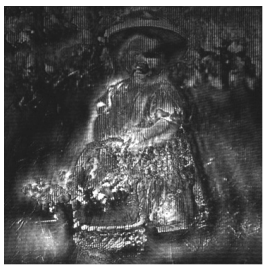

(d)

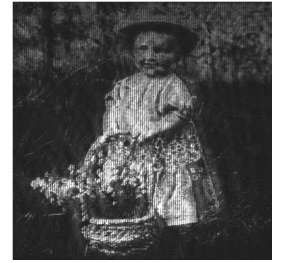

(b)

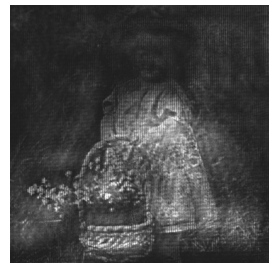

(e)

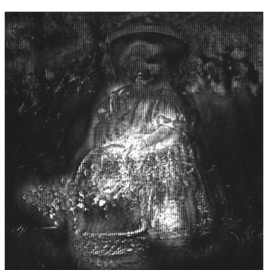

(c)

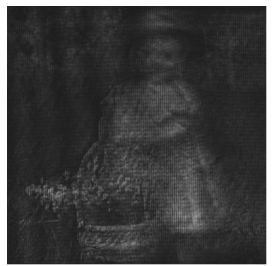

FIG. 7. Experimental results. Output intensity images with the phase shifted photopolymer corresponding to the same energy but different illuminating beam intensity and the exposure times: (a) $I=0.51 \mathrm{~mW} / \mathrm{cm}^{2}$ with 11.5 minutes exposure time, (b) $I=0.74 \mathrm{~mW} / \mathrm{cm}^{2}$ with 8 minutes exposure time, (c) $I=0.98 \mathrm{~mW} / \mathrm{cm}^{2}$ with 6 minutes exposure time, (d) $I=1.25 \mathrm{~mW} / \mathrm{cm}^{2}$ with 5 minutes exposure time, (e) $I=1.78 \mathrm{~mW} / \mathrm{cm}^{2}$ with 3.5 minutes exposure time, and (f) $I=2.23 \mathrm{~mW} / \mathrm{cm}^{2}$ with 2.5 minutes exposure time.

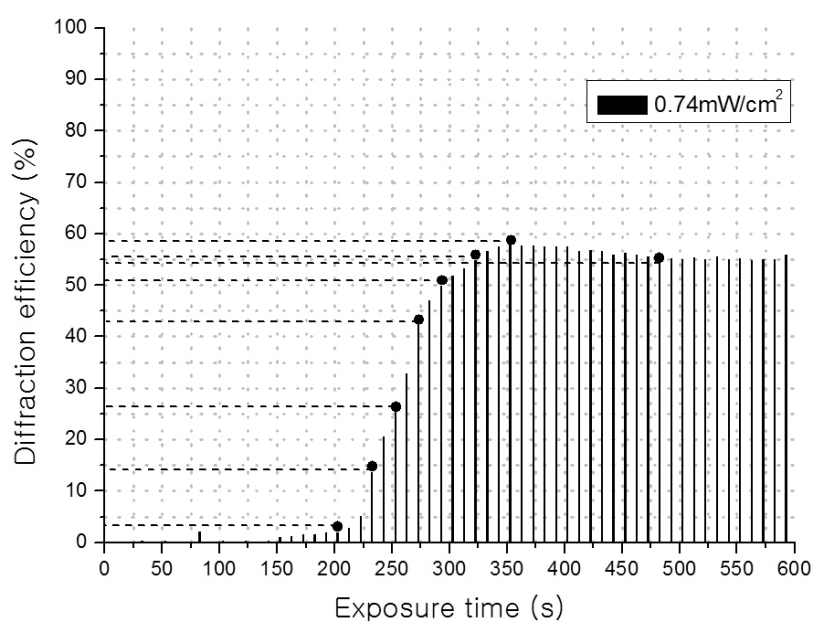

FIG. 8. The variation of diffraction efficiency of photopolymer according to the exposure time with fixed beam intensity $I=0.74 \mathrm{~mW} / \mathrm{cm}^{2}$.

example, the energy in case of $I=0.74 \mathrm{~mW} / \mathrm{cm}^{2}$ with 8 minute exposure time and $I=0.51 \mathrm{~mW} / \mathrm{cm}^{2}$ with 11.5 minute exposure time is the same. Figure 7 reveals that the quality of the output intensity image varies significantly even though we use the illumination of the same energy in implementing the phase contrast filter using the photopolymer. In fact, this is the natural consequence of the characteristics of the photopolymer. As shown in Fig. 2, the refractive index change does not depend

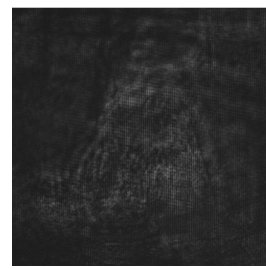

(a)

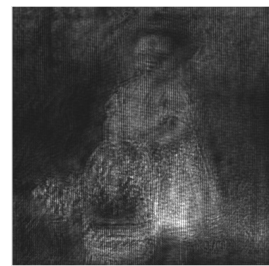

(d)

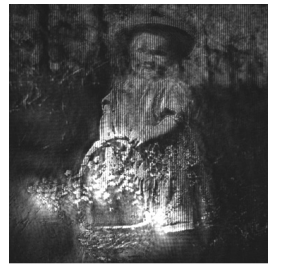

(g)

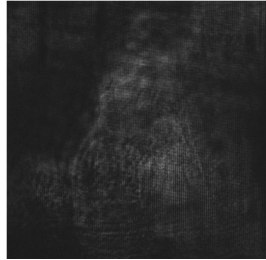

(b)

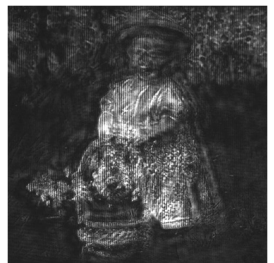

(e)

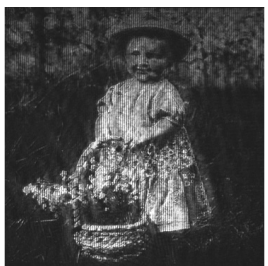

(h)

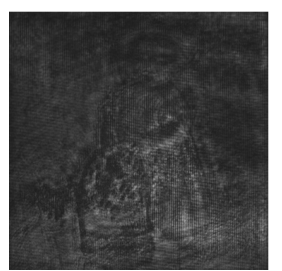

(c)

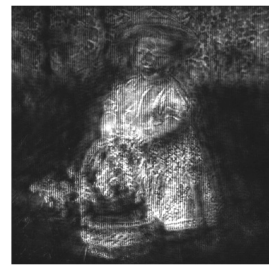

(f)
FIG. 9. Experimental results. Output intensity images with the phase shifted photopolymer with $I=0.74 \mathrm{~mW} / \mathrm{cm}^{2}$ and different exposure times for: (a) 3.5 minutes, (b) 3.8 minutes, (c) 4.3 minutes, (d) 4.6 minutes, (e) 4.9 minutes, (f) 5.4 minutes, (g) 5.8 minutes, and (h) 8 minutes.

on the illumination energy but depends on the illuminating beam intensity and the exposure time, individually. Therefore it is critical to keep the optimal beam intensity and the exposure time to get the desired refractive index change and consequently high quality output intensity image. Figure 7 confirms this point.

Next, we investigate the variation of the output intensity image quality according to different exposure time. The beam intensity is fixed at a value $I=0.74 \mathrm{~mW} / \mathrm{cm}^{2}$. In experiment, eight different exposure times are used as marked in Fig. 8.

Figure 9(a)-9(h) show the output intensity images. From Fig. 9. we can see that as the exposure time increases the amount of the induced refractive index change also increase, resulting in higher quality output intensity image. Note that at this beam intensity $I=0.74 \mathrm{~mW} / \mathrm{cm}^{2}$ the diffraction efficiency saturates to $55 \%$ as shown in Fig. 2 or Fig. 8, which can be interpreted such that induced refractive index change saturates to optimum value $\Delta n=0.0035$, generating desired $\frac{\pi}{2}$ phase shift. Therefore as the exposure time increases, the output intensity image quality is expected to be enhanced, and the results shown in Fig. 9 confirm this point. In a short summary, Figs. 6, 7 and 9 show that the beam 
intensity and the exposure time are the key parameters in implementing the phase contrast filter using the photopolymer.

The conversion efficiency, which is defined by the intensity ratio between the phase modulated image and converted intensity as shown in Fig. 4(b) is measured to be $82 \%$ in the optimal condition, i.e. $\Delta n=0.0035$. This indicates that if we have an ideal phase-type SLM which modulates the phase without any loss, we can implement a projection display of $82 \%$ light efficiency, which can not be achievable by the amplitude type projection display in principle.

\section{SUMMARY}

We have shown that it is possible to construct a phase contrast projection display system using photopolymer as phase contrast filter. Our experiments include 2 steps: first, make phase shift $\frac{\pi}{2}$ at a central point of photopolymer by focused illumination; second, realize phase contrast projection display using the phase shifted photopolymer. The beam intensity is adjusted in two steps properly. The experimental result shows the successful conversion of the phase image into the intensity image by the photopolymer, hence confirming the feasibility of the proposed method. We also demonstrated that the illuminating beam intensity and the exposure time in the fabrication process of the phase contrast filter using photopolymer are critical for the optimal performance. Although the phase contrast projection display using the photopolymer shows good performance, it has to be mentioned that there are several limitations originating from the use of the photopolymer. Because our experiment includes two steps, i.e. phase contrast filter fabrication, and projection display, it takes several minutes to obtain the proper phase shifted photopolymer before we proceed to the projection display step. In addition, the long time stability of the photopolymer has to be enhanced for a more reliable system. Therefore, further research is required to improve these points.

\section{ACKNOWLEDGMENT}

"This research was supported by the MKE (Ministry of Knowledge Economy), Korea under the ITRC (Information Technolgy Research Center) Support program supervised by the IITA (Institute of Information Technology Advancement).” (IITA-2008-C1090-0801-0018)

\section{REFERENCES}

[1] J. W. Goodman, Introduction to Fourier Optics, $2^{\text {nd }}$ ed. (McGraw-Hill, San Francisco, Calif., 1996), pp. 220-222.

[2] F. Zernike, "How I discovered phase contrast," Science, vol. 121, no. 3141, pp. 345-349, 1955.

[3] J. Glückstad, “Adaptive array illumination and structured light generated by spatial zero-order self-phase modulation in a Kerr medium," Opt. Comm., vol. 120, pp. 194-203, 1995.

[4] P. C. Mogensen and J. Glückstad, "Phase-only optical encryption,” Opt. Lett., vol. 25, pp. 566-568, 2000.

[5] V. R. Daria, P. J. Rodrigo, S. Sinzinger, and J. Glückstad, "Phase only optical decryption in a planar-integrated micro-optics system,” Opt. Eng., vol. 43, pp. 2223-2227, 2004.

[6] J. Glückstad, D. Palima, P. J. Rodrigo, and C. A. Alonzo, "Laser Projection using generalized phase contrast," Opt. Lett., vol. 32, no. 22, pp. 3281-3283, 2007.

[7] C. A. Alonzo, P. J. Rodrigo, and J. Glückstad, "Photonefficient grey-level image projection by the generalized phase contrast method," New J. Phys., vol. 9, pp. 132146, 2007.

[8] J. Liu, J. Xu, G. Zhang, and S. Liu, "Phase contrast using photorefractive LiNbO3: Fe crystals," Appl. Opt., vol. 34, no. 22, pp. 4972-4975, 1995.

[9] K. Sendhil, C. Vijayan, and M. P. Kothiyal, "Spatial phase filtering with a porphyrin derivative as phase filter in an optical image processor," Opt. Comm., vol. 251, pp. 292-298, 2005.

[10] M. L. Piao, N. Kim, J. H. Park, C. W. Shin, and S. G. Gil, "Realization of phase contrast filter using photopolymer," In The $15^{\text {th }}$ Conference on Optoelectronics and Optical Communications, Proc. COOC, vol. 15, no 1, pp. 435-436, 2008.

[11] D. Palima and V. R. Daria, "Holographic projection of arbitrary light patterns with a suppressed zero-order beam," Appl. Opt., vol. 46, no. 20, pp. 4197-4201, 2007.

[12] D. Palima and J. Glückstad, "Comparison of generalized phase contrast and computer generated holography for laser image projection," Opt. Exp., vol. 16, no. 8, pp. 5338-5349, 2008.

[13] V. Moreau, Y. Renotte, and Y. Lion, "Characterization of DuPont photopolymer: determination of kinetic parameters in a diffusion model," Appl. Opt., vol. 41, no. 17, pp. 3427-3435, 2002.

[14] Q. Huang and P. R. Ashley, "Holographic Bragg grating input-output couplers for polymer waveguides at an 850-nm wavelength, " Appl. Opt., vol. 36, no. 6, pp. 1198-1203, 1997.

[15] N. Kim, E. S. Hwang, and C. W. Shin, "Analysis of optical properties with Photopolymers for holographic application," J. Opt. Soc. Korea, vol. 10, no. 1, pp. 1-10, 2006 .

[16] H. S. Jeong, N. Kim, J. S. Yun, T. H. Park, and C. W. Shin, "Optical characteristic and image recording of reflection type Photopolymer in transmission structure," J. Opt. Soc. Korea, vol. 18, no. 1, pp. 8-13, 2007. 